\title{
La remunicipalización del servicio de abastecimiento urbano de agua: instituciones y común
}

\author{
Edurne Bagué Tova ${ }^{1}$
}

Recibido: 25 de marzo de 2017 / Aceptado: 24 de abril de 2017

Resumen. En el contexto social y político catalán surgido tras el estallido de la crisis de finales de 2008, el debate sobre la gestión del agua ha ido tomando relevancia pública hasta el punto de ser parte central en los programas políticos que las nuevas candidaturas electorales surgidas después del movimiento conocido como $15 \mathrm{M}$ prepararon para las elecciones municipales de 2015. Este proceso ha ido en paralelo con los cambios sociales acontecidos después del 15 de mayo de 2011, hecho que se constata en distintos aspectos. Algunas de las características que marcan este periodo son los cambios en el discurso, el cambio de algunos de sus protagonistas, el trato de la gestión del agua; así como la sustitución progresiva de una estrategia centrada en la no privatización, hacia el reclamo del aumento de la capacidad de decidir de la ciudadanía en la gestión del agua en el ámbito municipal. Pasando de una lucha a escala estatal y/o autonómica a una a escala local. En este artículo se enumeran aquellos aspectos que dan pie a la pregunta: ¿Qué concepción del común articula el nuevo escenario de lucha por el agua municipal y qué formas empíricas adquiere?

Palabras clave: Remunicipalización; participación; instituciones; comunes.

\section{[en] The Remunicipalization of the Urban Water Supply Service: Institutions and Common}

\begin{abstract}
In the Catalan context that emerged with the outbreak of the crisis in the winter of 2008, the debate on water management has become important to the point where it is a central part of the political programs that the new candidacies that emerged after the square occupations in 2011 prepared for the local council elections of 2015. This process has run altogether with the social changes that took place after May 2011, with the citizen movement known as $15 \mathrm{M}$, a fact that is verified in different aspects, such as: the message, change of subjects and treatment of water; As well as an important shift of a strategy focused on non-privatization, towards a demand of the increase of the capacity of the citizens in taking decisions on water management at the municipal scope. Moving from a national and / or regional struggle to a local scale.

This article describes those aspects that give rise to the question: What conception of the common are listed articulates the new stage of struggle for municipal water and what empirical forms adopted.

Keywords: Remunicipalization; participation; institutions; commons.
\end{abstract}

Sumario. 0. Apuntes sobre la metodología. 1. La situación de la gestión municipal del agua en Catalunya. 2. Mirada histórica de la gestión del servicio de abastecimiento de agua de las ciudades. 3. Los puntos negros de la gestión del servicio de abastecimiento. 3.1. Transparencia. 3.2. Déficit democrático. 3.3. Derecho Humano al agua. 4. Aproximarse a la remunicipalización. 5. ¿Es posible hablar de la apertura hacia un nuevo marco? 6. A modo de cierre. 7. Bibliografía 
Cómo citar: Bagué Tova, E. (2017). La remunicipalización del servicio de abastecimiento urbano de agua: instituciones y común, en Revista de Antropología Social 26(2), 427-448.

\section{Apuntes sobre la metodología}

El presente artículo analiza el proceso de remunicipalización del servicio de abastecimiento urbano de agua en la ciudad de Terrassa (Barcelona). Para ello, el trabajo se sitúa en el marco de la investigación-acción, que en este caso cuenta con dos fases diferenciadas. Una primera relacionada con la participación en la plataforma Aigua és Vida ${ }^{2}$ desde finales de 2010, en el grupo de Barcelona y posteriormente en el de Girona durante los años 2013 y 2014 —momento en el cual empieza la conceptualización del problema de investigación-. Y una segunda fase de trabajo etnográfico que se traduce en la sistematización de los registros y los datos, y que se lleva a cabo con el grupo de personas que conforman la Taula de l'aigua de Terrassa, mayoritariamente vecinos y vecinas de la ciudad vallesana que mantienen su autonomía respecto a las distintas fuerzas políticas. Como explican, en tanto que grupo de ciudadanos/as, tienen objetivos que no siempre coinciden con los de las fuerzas políticas.

El seguimiento desde las organizaciones ha permitido tener acceso a la documentación generada en estos años, desde materiales de difusión a la guía para la remunicipalización Per on començar? Guia pràctica per caminar cap a una gestió no mercantil i democràtica de l'aigua creada por Aigua és Vida. A la par, se ha tenido acceso a los debates, organización de actividades, movilizaciones y reuniones de trabajo, jornadas y talleres que se han llevado a cabo en el transcurso de estos años ${ }^{3}$. En este contexto, la investigación-acción se mostraba como una herramienta útil, pues la posición que la investigadora ocupa en relación al tema y los actores - acceso y proximidad a espacios de reflexión y conversaciones - ha permitido establecer una relación de reciprocidad, o "retorno". Según Fals Borda (1986) uno de los elementos más relevantes de la investigación-acción es el de la transformación social como uno de los principios que impulsan esta metodología, porque de él derivan otros aspectos definitorios y característicos como la "devolución" o el "retorno". Por lo tanto, la devolución es una forma de cerrar el círculo que vincula la metodología usada con el objetivo de la transformación social. Por eso, otro de los aspectos que definen la investigación-acción es el diálogo constante entre praxis e investigación, a partir del cual se avanza en la construcción de alternativas y propuestas. En el caso que nos ocupa, lo que se denomina como "movimiento del agua" tiene una base estructural consolidada en este sentido, puesto que distintos espacios de investigación y/o gestión sobre la materia forman parte de estos espacios de movilización, como son

\footnotetext{
Según exponen en su página web, Aigua és Vida es una plataforma formada por organizaciones de la sociedad civil. En la misma se pueden consultar los mapas del agua. Para la elaboración de este artículo fue consultada en mayo 2015.

3 Se enumeran aquí las actividades llevadas a cabo durante el último trimestre de 2016: Encuentro de Ciudades por el agua Pública que tuvo lugar en Madrid en noviembre de 2016, la Jornada de Remunicipalización organizada por el Ajuntament de Barcelona en diciembre del mismo año, el Taller Internacional sobre participación en la gestión del servicio de abastecimiento domiciliario que tuvo lugar en Terrassa, el debate con las experiencias de París, Nápoles, Córdoba y Olesa de Montserrat o el taller Derecho Humano al Agua y Tarifas, entre otras.
} 
$\mathrm{AEOPAS}^{4}$ o la $\mathrm{FNCA}^{5}$. En el caso del artículo que aquí se presenta, esta trayectoria por los distintos grupos ${ }^{6}$ da cuenta de una visión que queda reflejada en el texto y en la exposición de los elementos, que parten de las reflexiones, análisis, conversaciones, explicaciones y posicionamientos que estos grupos se hacen.

Por otro lado, el artículo también cuenta con otra fuente de información que viene a completar la parte del trabajo etnográfico. Hablamos de más de diez entrevistas en profundidad a distintas personas pertenecientes a dichos espacios, desde Aigua és Vida Barcelona y Girona, como Taula de l'aigua de Terrassa. Y, por último, van acompañadas también de otras fuentes como el acceso a memorias, informes, expedientes de distintos casos vinculados a la temática y un aporte de fuentes de hemeroteca. Todo ello ha contribuido a tener una sólida base de elementos a partir de los cuales establecer el análisis que aquí se presenta.

\section{La situación de la gestión municipal del agua en Catalunya}

El desmantelamiento del Estado del Bienestar en el Estado español y Catalunya se caracteriza por la progresiva privatización de los servicios sociales a través de reformas estructurales que se han traducido en recortes en los servicios públicos de ámbito social, como la educación o la sanidad, en la precarización de los derechos laborales y los salarios mediante la aprobación de sucesivas reformas laborales (Cebollada, 2014), que unidas a la crisis financiera, han contribuido al aumento del desempleo. Ante este escenario se pueden observar dos tendencias generales. Mientras que un sector de la población está optando por un aumento de actitudes relacionadas con el marco neoliberal de acción, esto es, una apuesta hacia conductas de corte netamente individualista, otra parte tiende a la articulación de redes de apoyo y economía social ${ }^{7}$, organización y gestión de los servicios básicos de distinta índole. En este segundo grupo es donde se ubica la recuperación de la gestión del servicio de abastecimiento domiciliario de agua de las poblaciones por parte del organismo público competente.

En su definición en el diccionario de la Real Academia Española de la Lengua, municipalizar es "convertir en municipal un servicio público que estaba a cargo de una empresa privada" y en el diccionario del Institut d'Estudis Catalans consta como "transferir la propiedad o empresa (de un servicio) al municipio". Podemos advertir que la definición se ciñe a "quién" adquiere la responsabilidad, pero no se describe el "cómo" se llevará a cabo el desarrollo de dicha actividad. Sin embargo, para las personas que fundan grupos que luchan y reivindican la remunicipalización, como la Taula de l'Aigua en Terrassa, el "cómo" y el "quién" tienen la misma importancia.

\footnotetext{
Asociación Española de Operadores Públicos de Abastecimiento y Saneamiento.

Fundación de la Nueva Cultura del Agua.

Aunque el presente artículo parte de tres fuentes directas de información: Aigua és Vida Catalunya, Aigua és Vida Girona y la Taula de l'aigua de Terrassa; también se hacen referencias a otros grupos existentes en otras poblaciones, tanto catalanas como del resto del Estado español. Sin embargo, no hay un registro de los grupos que existen; algunos empiezan y se desactivan, otros continúan su labor, otros pasan por momentos distintos en intensidad de trabajo, puesto que principalmente dependen del tiempo que pueden dedicar debido a su carácter voluntario y activista.

Algunos ejemplos de redes de apoyo vinculados a la economía social son la Plataforma Afectados por la Hipoteca y la Xarxa Economia Solidaria (XES) que aglutina la mayoría de las iniciativas de este sector (http://www. xes.cat/pages/xs100.php)
} 
Para estos grupos, el objetivo va más allá de la recuperación de la gestión pública, promueven el pensamiento de un nuevo modelo de gestión del agua municipal que incluya a la ciudadanía como sujeto activo.

Entre las experiencias catalanas recientes de recuperación de la gestión del servicio de abastecimiento domiciliario encontramos los municipios de Figaró-Montmany y Arenys de Munt. En ambos casos se trata de poblaciones pequeñas ${ }^{8}$. A pesar de la existencia de estas experiencias, el interés por trascender las formas actuales de gestión se ha puesto de relieve sobre todo de la mano de grupos como la Taula de l'aigua ${ }^{9}$ de Terrassa surgidos en los años sucesivos a la experiencia que supuso el $15 \mathrm{M}^{10}$.

Para hablar de gestión municipal del servicio de abastecimiento es imprescindible comprender los elementos básicos de la organización, la regulación y las competencias del lugar concreto, puesto que es muy diferente dependiendo de cada país. En el caso de España, la regulación específica en materia de agua se da en un marco estatal y en un marco autonómico, que están en estrecha relación y a partir del cual se establecen las competencias y los instrumentos de gobierno ${ }^{11}$. Así, en el caso de Catalunya, el Estatut d'Autonomia recoge las competencias en materia de agua que han sido transferidas desde el Estado español, por lo que las mismas están sujetas a lo que establezca la Constitución Española y la Directiva Marco del Agua ${ }^{12}$.

En esta materia el marco de regulación del Estado español, establece dos niveles de gestión: en alta y en baja. La gestión en alta comprende los tramos e infraestructuras que van desde el punto de captación a los puntos de conexión con los municipios. Esta competencia depende del gobierno autonómico, que en el caso de Catalunya es el Departament de Territori i Sostenibilitat a través de la Agència Catalana de l'Aigua (ACA). Dentro de la gestión en alta queda la planificación, ordenamiento de las infraestructuras necesarias a nivel de territorio, tales como trasvases y grandes infraestructuras ${ }^{13}$. Luego está la gestión en baja, que es la que comprende el tramo desde el punto de conexión y la distribución por la red municipal de abastecimiento, y va a cargo del municipio ${ }^{14}$.

8 Arenys de Munt: 8.736 habitantes en 2015 y 1.094 en el Figaró-Montmany. Fuente: Institut d'Estadística de Catalunya (IDESCAT). Por sus perfiles, las mismas han entrado a formar parte de la CONGIAC, el consorcio de operadores públicos de Catalunya.

9 Información procedente de la observación en campo y de la Mini Guia de remunicipalización que se editó como herramienta para que los distintos grupos locales pudieran emprender dichos procesos. Aigua és Vida, como plataforma paraguas que concentra a todos los grupos locales existentes en Catalunya, en los primeros años ha centrado sus objetivos en la recuperación de la gestión, y no es hasta el año 2016, que ha abierto el espacio y el debate en torno a la reflexión y creación de la parte que atañe al control social y la participación de la ciudadanía en la toma de decisiones. Sin embargo, para la Taula de l'aigua la participación y control social ciudadano han sido siempre la prioridad

10 En la explicación de su origen evocan al 15M y el surgimiento del Procés Constituent en lo que encontraron ingredientes para una transformación profunda de la sociedad.

11 El marco viene dado por la Constitución Española, el Estatut d’Autonomia, la Ley Base de Régimen Local (LBRL), la Ley de Racionalidad Sostenibilidad de la Administración Pública (LRSAL) y la Ley Orgánica Estabilidad Presupuestaria y Sostenibilidad Financiera LOEPSF).

12 Directiva 2000/60/CE del Parlamento Europeo y el Consejo de 23 de octubre de 2000, por la que se establece un marco comunitario de actuación en el ámbito de la política de aguas que afecta a la legislación de los recursos hídricos en el marco español.

13 Expuesto en la web de la Agència Catalana de l'Aigua

14 La organización en la práctica es más compleja porque los municipios pequeños crean acuerdos de distinta índole para compartir gastos, gestión o compartir tramos de infraestructura. Este hecho conlleva la existencia de dos grupos de operadores públicos, AEOPAS en España y CONGIAC en Catalunya. También existen casos de mancomunidad o consorcio. En la práctica encontramos gran complejidad puesto que los contratos no siempre pertenecen al mismo momento jurídico-histórico, hecho que se traduce en dificultades en establecer jerarquías jurídicas de los distintos contratos. En la gestión en alta, el trasvase más grande de Catalunya lo opera Aigües 
Si tomamos las cifras publicadas por la plataforma Aigua és Vida (figura 1), el $81 \%$ de la población catalana reside en ciudades y pueblos que tienen formas de gestión pública indirecta, mayoritariamente vinculadas a AGBAR del grupo Suez. Estos datos dan cuenta de que, en el contexto catalán, las formas de gestión privada y/o mixta son una práctica extendida, en la que sólo cerca de un $20 \%$ de la población vive en poblaciones bajo régimen de gestión pública directa. Una cifra que contrasta con el $70 \%$ de los municipios europeos" ${ }^{\prime 15}$.

\begin{tabular}{|l|l|}
\hline \multicolumn{2}{|c|}{ GESTIÓN del AGUA en Catalunya } \\
\hline Municipio & Población \\
\hline Pública: 506 & 1.235 .606 habitantes \\
\hline Privada: 394 y Mixta: 45 & 6.317.583 habitantes (mixta-privada) \\
\hline \multicolumn{2}{|c|}{ Población total Catalunya: 7.553.189 habitantes } \\
\hline
\end{tabular}

*Fuente Aigua és Vida, 2015.

Figura 1

Teniendo en cuenta que Suez, junto con Veolia y Bechtel, son los tres grandes grupos que gestionan el acceso al agua a nivel mundial, podemos decir que la gestión actual del servicio de abastecimiento tiene lugar desde una realidad de monopolio privado transnacional que acentúa una situación de opacidad gestora frente a la administración pública, que no tiene acceso a las fuentes y datos del servicio. Dicho con otras palabras, la administración pública - ayuntamiento- a pesar de ser la titular y máxima responsable, no tiene capacidad de control sobre este servicio básico. En este contexto aparece la remunicipalización en Catalunya, definida por Kishimoto (2015) como la expresión de la voluntad de asumir colectivamente la gestión de los servicios más básicos.

Por su lado, Castro (2005) o Aboites (1999; 2009) plantean que hay una relación entre el modo como se conciben y crean los modelos de gestión, infraestructura e intervención política mediante políticas públicas en materia de aguas, y los modelos políticos en los cuales surgen. De ahí que, por sus características, la gestión del abastecimiento de agua abre un espacio de análisis que busca dilucidar el alcance de posibles cambios más profundos que se puedan estar generando.

\section{Mirada histórica de la gestión del servicio de abastecimiento de agua de las ciudades}

En términos generales, el servicio básico de abastecimiento municipal aparece en la segunda mitad del siglo XVIII en ciudades como Londres o París, y se extiende de forma generalizada a principios del siglo XIX, coincidiendo con el impulso tecno-

del Ter Llobregat -ATLL Concessionària de la Generalitat de Catalunya, SA, una empresa pública, privatizada en 2012, aunque en 2015 el Tribunal Superior de Justicia de Catalunya, anuló la adjudicación por considerar que "el concurso para privatizar la empresa pública de agua de Barcelona vulneró "los principios de publicidad, concurrencia e igualdad".

15 Datos extraídos del artículo de eldiario.es "La municipalización del agua, una idea que se abre camino " 
lógico de la Revolución Industrial (Heredia, 2014) y su extrapolación de los conocimientos de la ingeniería aplicados a la mejora de los servicios urbanos. Hastrup (2013) apunta que con Linton se establece la idea de que el ciclo hidrológico es un hecho establecido, dando paso a una noción del agua como recurso abstracto que puede ser medido y contado.

En este contexto, la implementación aparece sobre todo de la mano de empresas privadas de tipo familiar, la mayoría de las veces vinculadas al impulso de la industria y que se encargan de ofrecer este servicio a las élites de las ciudades, sin responsabilidades en la calidad del servicio ${ }^{16}$. Sin embargo, como apunta Bakker (2010), estas iniciativas privadas se muestran incapaces de ampliar la cobertura a los barrios de rentas más bajas. Por su lado, el surgimiento del movimiento higienista, con incidencia en ciudades de Europa y Estados Unidos (Castro, 2005; Matés, 2016), impulsa la necesidad de garantizar el acceso al agua de calidad apta para el consumo humano con la finalidad de evitar epidemias. Estas garantías se plasman en la regulación para el control de la calidad y la infraestructura, de tal modo que el movimiento higienista marcó un punto de inflexión en muchas ciudades. La suma de un sector político de corte más universalista, desde el que se acuñan conceptos como «conciencia sanitaria» o «política social» (Castro, 2005), que promueven la asunción por parte de los gobiernos municipales de la gestión del servicio, y una reforma de la regulación de dicho servicio promovida por el movimiento higienista, confluye en una tendencia hacia la municipalización del servicio de abastecimiento básico, debido a la noción de responsabilidad social de dichos sectores políticos, y en tanto que la incorporación de las nuevas medidas desalentó al empresariado del momento (Matés, 2014).

En la actualidad, ciudades como Barcelona, Girona o Terrassa, tienen su servicio de abastecimiento operado por empresas pertenecientes a AGBAR, del grupo francés Suez. Al menos Barcelona y Terrassa tienen trayectoria histórica en este modelo de gestión privada que proviene de finales y mediados del siglo XIX. Un hecho que añade interés al fenómeno de la remunicipalización, puesto que la gestión estuvo bajo manos de los organismos públicos sólo durante el período de la Guerra Civil (Gorostiza, March y Sauri, 2012; Marcet, 1986), lo que nos indica que la remunicipalización no se ciñe a la recuperación de la gestión del servicio previamente privatizado.

\section{Los puntos negros de la gestión del servicio de abastecimiento.}

Dentro del marco legal de gestión de servicios básicos existen las formas de gestión directa e indirecta para el servicio de abastecimiento domiciliario. Dentro de la gestión indirecta está la concesión a una empresa privada o la constitución de empresas de participación pública-privada (PPP) ${ }^{17}$, ambas practicadas extensivamente en $\mathrm{Ca}$ talunya.

Delclòs (2008) señala que la promoción de las PPP procedía del mismo sector privado que buscaba así reducir el riesgo de inversión a la vez que diluía responsa-

16 Como señala Bakker (2010), aunque la ejecución de las redes de alcantarillado y distribución fue llevada a cabo de forma mayoritaria por la iniciativa privada, una parte también lo fue desde los gobiernos municipales.

17 LBRL, artículo 85, que ha sido modificado cualitativamente desde su creación en 1985. 
bilidades ${ }^{18}$. Dentro de este formato, la repartición de los porcentajes varía en función del caso, aunque la mayoría de las veces los porcentajes de participación accionarial del sector público son inferiores de aquellos que provienen del sector privado, de tal modo que el control sobre la gestión recae en el sector privado. Así sucede en Girona, donde el $20 \%$ de las acciones ${ }^{19}$ corresponden a las tres ciudades adscritas mientras el $80 \%$ restante pertenece a la empresa privada Girona S.A.

Bajo las formas vigentes de gestión, los usuarios pasan a ser tratados como clientes, y esto repercute en la relación entre los actores, puesto que, dentro de este esquema, no se contempla que haya necesidad de comunicación entre clientes y responsables del servicio. Este punto es importante porque supone la nulidad de cualquier derecho que la ciudadanía pudiera ejercer como tal en tanto que, al quedar fuera, no puede reclamar ninguna obligación o responsabilidad más allá de la propia entre consumidor-empresa. El diálogo sobre la gestión queda entre el ente público y la empresa (como proveedora del servicio). Es dentro de este modelo que se sitúan los argumentos expuestos por espacios como la Taula de l'Aigua acerca de su interés en ser reconocidos como ciudadanía en un marco de gestión pública directa.

Para las personas que forman parte de estas plataformas locales, el modelo actual de gestión del servicio de abastecimiento adolece principalmente de ser un monopolio transnacional ${ }^{20}$, con gran afluencia de puertas giratorias y relaciones clientelares con políticos de ciertos partidos, con una gran falta de transparencia y un déficit democrático estructural que favorece la vulneración del Derecho Humano al agua, por su gestión en torno a criterios de racionalidad económica donde el agua es entendida como una mercancía.

\title{
3.1. Transparencia
}

\begin{abstract}
"Yo creo que ahora, en este momento, la percepción de la ciudadanía es que tiene que haber transparencia. Pero hace unos años, cuando esto pasaba, no era así. Hemos cambiado. Parecía que, si tu votabas a un grupo de políticos y estos tenían la mayoría, lo gestionaban, y al cabo de cuatro años, si a ti te iba bien como lo habían hecho (los volvías a votar) y se hacía de esta manera. Nunca, en general, ha habido la intención de ser transparentes y explicarlo todo. Ahora sí porque la ciudadanía
\end{abstract}

18 Actualmente en el proceso que se vive en la ciudad de Terrassa, con motivo del fin de la concesión del año 1941, la concesionaria, Mina Pública de Terrassa S.A., ha propuesto al ayuntamiento la constitución de una sociedad mixta. La idea es ofrecer al ayuntamiento un mecanismo que le permita no endeudarse, a través de un contrato con la duración necesaria para hacer efectiva la amortización de los activos en litigio. De esta manera, la actual concesionaria accede a una renovación de la concesión sin pasar por un concurso al que se podrían presentar empresas de otros grupos. Esta estrategia es la misma que se llevó a cabo en la constitución de la Sociedad Mixta del Área Metropolitana de Barcelona y que fue declarada nula por el TSJC.

$1914 \%$ Ayuntamiento de Girona, 4\% Ayuntamiento de Salt y el 2\% Ayuntamiento de Sarrià de Ter. Girona S.A. Con el $80 \%$ se divide en Caixabank 34,22\%, Aqualia 33,61\%, Agbar 31, 42\% y un 0,75\% de pequeños accionistas locales (familias importantes de la zona).

20 Cabe destacar que, a pesar de la tradición existente en algunas ciudades catalanas por una gestión realizada mediante concesionario privado, los mismos han cambiado considerablemente desde los orígenes del servicio a la actualidad, puesto que ahora, lejos de tratarse de iniciativas privadas de carácter local, se trata de empresas pertenecientes a estructuras oligopólicas de capital transnacional. Este hecho está relacionado con los cambios de modelo económico de espectro más amplio acontecidos en los últimos siglos. 
está pidiendo participar directamente en la toma de decisiones, y más en este nivel, con cosas que nos afectan directamente.” (V.S. Girona, abril 2015)

Uno de los elementos detectados y estrechamente vinculado al déficit democrático es la falta de transparencia. Cuando el servicio es operado por una empresa privada no hay acceso a datos duros y primarios por parte de la corporación municipal. En Terrassa, la empresa que actualmente opera el servicio, Mina Pública d'Aigües S.A, no facilitó la información sobre la gestión que el ayuntamiento requería, llegando a bloquear la entrada de los técnicos del ayuntamiento a las oficinas de la empresa concesionaria ${ }^{21}$.

Un segundo factor que favorece la falta de transparencia es la ausencia de representación directa de la ciudadanía local en los espacios de toma de decisiones. Este aspecto determina la dimensión democrática de la gestión y enlaza la transparencia estructural con el precepto del control social ciudadano. En este punto detectamos uno de los pilares que sustentan la necesidad de un cambio de modelo. La introducción de este elemento en la gestión supone un antes y un después en las formas de operar de este servicio, ya que no estaba incorporado y apenas era tenido en cuenta hasta ahora.

Finalmente, de forma independiente a los demás aspectos ya señalados, hay que incluir el acceso a la información ${ }^{22}$, pero también que ésta sea publicada con lenguaje y formatos comprensibles a la mayoría de la población, ya que actualmente este tipo de información no siempre se publica, y aun cuando se hace, no siempre usa medios comunicativos accesibles y comprensibles a la mayoría de la ciudadanía; por lo tanto, a efectos prácticos, se traduce en una situación de desinformación.

Como se ha ido señalando, estas deficiencias relacionadas con la transparencia no son exclusivas de los operadores privados, también se dan situaciones parecidas bajo régimen de gestión pública, lo cual nos lleva al epicentro del diagnóstico sobre la necesidad de un cambio de modelo en la gestión. No basta con que ésta recaiga en una titularidad y una gestión pública, es necesario que permita a la ciudadanía tener mecanismos legales a su disposición para presionar y cumplir con sus labores de vigilancia. Por este motivo se habla de gestión directa y con control social ciudadano.

Cabe destacar que no existe un modelo cerrado y definido, lo que existen son unas líneas básicas compartidas por las experiencias de remunicipalización, como la emprendida en la ciudad de París, símbolo de la remunicipalización y que sirve de orientación $^{23}$.

\subsection{Déficit democrático}

El punto de la transparencia nos lleva a otro planteamiento hecho por las personas que constituyen estos grupos: el del déficit democrático en la gestión del servicio de abastecimiento de agua urbana. Éste se centra principalmente en el hecho de que

21 "Terrassa podria dur Mina als tribunals per haver impedit la inspecció d'instal·lacions"; el artículo fue publicado en El Punt Avui el día 26 de noviembre de 2016.

22 Tal y como establece el Convenio de Aarhus y su transposición a la legislación española.

23 En el momento en que se redacta el artículo se está en proceso de construcción una propuesta de modelo de gestión urbana del agua. Para ello, se han revitalizado los contactos de los distintos grupos que trabajan en distintas poblaciones del Estado Español, con el afán de poner en común las líneas básicas compartidas y está a punto de salir a la luz un documento de conclusiones del Taller Internacional de Ciudadanía y Gestión del Agua: ¿Cómo implicamos activamente a la sociedad en la gestión del agua?, que tuvo lugar en Terrassa el 22 de octubre de 2016. 
la ciudadanía no tiene vías para acceder a la información, ni mecanismos de participación social activa y directa. Esto se agudiza en el modelo actual predominante de concentración, donde la mayoría de las empresas están operadas por empresas filiales de $\mathrm{Suez}^{24}$, lo cual consolida una estructura en la que los objetivos del grupo empresarial y los criterios para la toma de decisiones son ajenos al lugar donde impactan. Esto favorece la disminución de espacios de ejercicio democrático vinculados al territorio y al municipio.

A pesar de que el origen del servicio domiciliario está vinculado a las empresas privadas en los primeros años de su existencia como tal, la tendencia ha derivado en una situación de monopolio a gran escala. Durante el siglo XIX, tanto si había una o más empresas operando el servicio, éste tenía alcance local, puesto que generalmente se trataba de empresas familiares y locales (Matés, 2016). Sin embargo, este escenario ha cambiado de forma vertiginosa, de tal modo que los mismos grupos empresariales (Suez, Veolia y Bechtel), con diferentes nombres, han obtenido las concesiones para gestionar cada vez más fases del ciclo del servicio urbano de agua. También incluimos todos los servicios asociados como la construcción de plantas de tratamiento de aguas residuales, laboratorios de potabilización, laboratorios de análisis de control de calidad del agua, el mantenimiento de la red y de las infraestructuras.

A esta situación, se añade que a los nuevos contratos de concesión se les incorpora, mediante cláusulas, el cumplimiento de los Bilateral Investment Treaties (BIT) descritos de forma precisa y técnica por Kishimoto (2015). La vinculación que se establece a estos tratados y el hecho de que los operadores privados son grandes grupos transnacionales, contribuyen a vaciar y anular las competencias y potestades atribuidas a las instituciones públicas y mueven la gestión del servicio de abastecimiento de agua hacia el marco legal del derecho mercantil.

La falta de conocimiento experto es usada para justificar que los ciudadanos no cuenten con la capacidad (conocimiento) suficiente sobre la materia. Según Delclòs (2008), esta es la vieja idea frente a la cual la sociedad civil propone un modelo público de gestión directa del agua que incluya la participación y el control social.

\subsection{Derecho Humano al agua}

Ante la situación descrita, el Derecho Humano al agua ha quedado seriamente afectado. Una circunstancia frente a la cual diferentes grupos y plataformas han emprendido acciones de protesta, en particular cuando ha habido un aumento de las tarifas, que según recoge el preámbulo de la Ley 24/2015, superó el $60 \%{ }^{25}$ entre los años 2008 y 2013. Esto conllevó cortes de suministros a familias que no pudieron afrontar el pago de los servicios, situaciones muchas veces asociadas a los desahucios por impago de las hipotecas. Este hecho abrió el debate en torno al Derecho Humano y la Pobreza Energética. A la luz de lo expuesto, las personas que están luchando por el agua, detectan que tanto los cortes de suministro, el aumento de tarifas, como el poco interés en dar un buen servicio se relacionan con el aumento del margen de beneficios, objetivo

24 La situación de monopolio transnacional se ha hecho pública en los últimos años, pero hasta el momento se daba sin más repercusión.

25 El Preámbulo de la Ley 24/2015 señala que en Catalunya y desde el año 2008, el precio de la luz se ha incrementado en un $60 \%$ y el del agua un 66\%, según cita el Informe Pobresa i privació de subministraments bàsics. 'Pobresa energètica' a Catalunya, una qüestió d'ingressos publicado en noviembre de 2016. 
incompatible con el del interés público general propio de la prestación de servicios básicos, y más si tiene consideración de Derecho Humano. Los propios grupos se anclan en el Derecho Humano al agua para la exigencia de transparencia, el derecho a la información o el establecimiento de la rendición de cuentas, acercando la noción de dicho derecho a una noción de derecho económico, social y cultural. También en este tema se observa que el debate está incorporando nuevos argumentos según los cuales el cambio debe darse en el modelo de gestión y no solamente en la titularidad de la misma. Esto es debido a los perjuicios que causa un modelo de gestión pública en el que predomina la lógica empresarial (McDonald, 2015).

Como ya se ha comentado, la relación que se establece desde la empresa con la ciudadanía es de empresa-cliente, de ahí que la empresa pueda ejercer su presión practicando cortes de suministro por falta de pago en una transformación del espacio de la política pública en ámbito mercantil. En este punto, los grupos que reivindican el agua y su gestión pública vinculan este fenómeno con un atentado al principio del derecho a la vida misma e instan a la administración pública a comprometerse en dar respuesta a este tipo de situaciones ${ }^{26}$.

Por eso McDonald (2015) destaca que los políticos deberán pensar más que nunca en el significado de lo público. Vemos con esta aseveración que, a través de la remunicipalización, lo que está en debate es la relación y la concepción de lo público y lo común: cómo esto se traduciría en nueva institucionalidad a través de la cual se llegaría a los principios y objetivos de las políticas públicas que tienen que venir, y donde entra —o debería entrar - la ciudadanía como elemento principal en la toma de decisiones. Es decir, como elemento estructural de un modelo en el que la ciudadanía forma parte de la toma de decisiones a dos niveles: primero, en aquellos aspectos asociados a la creación del modelo, a partir del cual se lleve a cabo la gestión en el futuro y de los espacios en los cuales se tomaran decisiones de distinto calibre (más estratégicas, ejecutivas o técnicas). Y, en un segundo nivel, como actores de igual peso y relevancia en la toma de decisiones cotidianas. En definitiva, lo que se está abriendo es un debate que puede trascender desde el nivel ontológico y epistemológico hacia el más material y cotidiano, acerca de qué se entiende por público y cuál es el alcance de las políticas públicas.

\section{Aproximarse a la remunicipalización}

Para Lobina (2015) la remunicipalización es un proceso de transición del servicio público de agua a una gestión y propiedad "pública plena", bajo control democrático, que a su vez ofrece la posibilidad de realizar ideas colectivas y una oportunidad para abastecer un sistema público socialmente interesante y ecológicamente sostenible. Mientras que McDonald (2015) destaca que la remunicipalización del agua como bien común le infiere una dimensión local inherente a partir de la cual la gestión

26 El Derecho Humano al agua, así como las distintas iniciativas que se están promoviendo en este sentido, son una puerta a un debate complejo lleno de contrariedades y de base profundamente epistemológica. En la actualidad se han habilitado distintas iniciativas para hacer frente a los cortes de suministro y así cumplir con el Derecho Humano al agua y garantizar el mínimo vital. La mayoría de las propuestas se centran en bolsas de ayuda que se ponen al servicio de los servicios sociales municipales que sirven para pagar las facturas que quedan impagadas por parte de abonados que no están en disposición de hacerlo. Estas soluciones tienen un carácter paliativo, pero no estructural y con ellas, las empresas siguen sin percibir las repercusiones de sus políticas tarifarias. 
no puede estandarizarse. Aparte, los autores citados coinciden en destacar que la remunicipalización tiene como objetivo desmercantilizar el agua, y así acercarla a su valor de uso y alejarla de su valor de cambio. De estas aportaciones se extrae que la remunicipalización adquiere una dimensión más compleja, política y social, que sobrepasa el ámbito estrictamente técnico, como la mera recuperación de la gestión del servicio por parte de la administración municipal. Implica cambiar los principios sobre los cuales esta gestión debe darse, abriendo la puerta a otras posibilidades.

Como se analiza en Our Public Water Future (Lobina et al. 2015) y en el informe Here To Stay. Water remunicipalisation as a global trend (Lobina et al. 2014), en quince años se han dado en el mundo 234 casos de remunicipalización, por eso los autores hablan de una "ola remunicipalizadora" (Lobina, 2016). En Catalunya encontramos entidades que tienen la problemática del agua como una de sus líneas de acción y trabajo desde la primera década del 2000, como es el caso de Enginyeria sense Fronteres, una de las entidades impulsoras de Aigua és Vida. En los encuentros internacionales que tuvieron lugar aquellos años se conformó una red internacional de grupos que luchaban por el Derecho Humano al agua y que buscaban poner de manifiesto aquellas problemáticas que no eran abordadas en los debates y foros oficiales, para así plantear otras maneras de gestionar los servicios básicos de agua y saneamiento (Delclòs, 2008).

En este período, la parte “de la municipalización quizás era un discurso más técnico e ideológico" "27 según nos cuenta uno de los impulsores de Aigua és Vida en Catalunya, quien explica que el origen de la plataforma se da cuando se estaban tramitando los expedientes de regulación en la Agencia Catalana de l'Aigua (ACA) y Aigües de Barcelona (AGBAR), y salió a la luz la sentencia de los juzgados de Barcelona que señalaba que "no había contrato de concesión" entre AGBAR y el Ayuntamiento de Barcelona ${ }^{28}$ (Balasch, 2012; Carrillo, 2015) para la gestión del servicio de abastecimiento ${ }^{29}$. En esa época, París recuperó la gestión del agua de la ciudad ${ }^{30}$.

En Catalunya se detecta un punto de inflexión entre la primera década del 2000 y después de 2011, momento en el que tiene lugar la proliferación de grupos locales, y el perfil de sus integrantes cambia. Antes de 2010 se trataba principalmente de personas con preparación técnica en la temática y que mediante las organizaciones no gubernamentales trabajaban desde una visión crítica sobre la gestión del agua, con articulación en la redes internacionales y foros alternativos. En cambio, a partir de 2012 se caracterizan por su carácter local y con un perfil heterogéneo, relacionado con la idiosincrasia social, histórica y política de cada población.

La aparición de nuevos grupos de carácter local se produce en 2012, lo cual hace que la temática vaya calando y más sectores de la ciudadanía se interesen en conocer

27 Entrevista a E.B. Barcelona, febrero de 2015

28 Información publicada en el portal de prensa Crític y en el Anuari dels Silencis Mediàtics. Todos los demás enlaces de la noticia han sido eliminados de la red y actualmente es difícil encontrar otras fuentes que traten este hecho.

29 Entrevista a Q.P. Barcelona, noviembre 2016. Durante la entrevista me comentaba que la noticia había desaparecido de internet y que sólo quedaba la pieza que aún existe en el portal del Crític y Anuari dels Silencis Mediàtics. Puedo certificar que lo que expone la persona entrevistada es cierto puesto que yo misma en ese momento seguía los acontecimientos y este hecho salió en distintos medios.

30 París no fue el único caso, en fechas cercanas también se recuperaron Grenoble, Berlín, entre otras y tuvo lugar el referéndum de Nápoles que abrió la puerta a la internalización del servicio de agua en esta ciudad. Pero París por su propia idiosincrasia: dimensión, sede central de Veolia y Suez, y por el cariz de la propuesta, cercana a las concepciones de la visión de ciclo del agua, se erigió como paradigma a seguir. 
cómo funciona la gestión del agua urbana. Este hecho conlleva la transversalización organizativa, y en poco tiempo Aigua és Vida se convierte en la red-paraguas que engloba todo el territorio a la vez que se erige como el espacio de referencia donde los distintos grupos comparten las experiencias y el conocimiento acumulado. Con la entrada en escena de estos nuevos grupos, personas sin experiencia ni conocimiento explícito en la materia se añaden y dan impulso a la remunicipalización.

En estos nuevos grupos podemos observar que la construcción del común se da a partir de la conciencia de un nosotros, que no parte de la suma de individualidades sino, en palabras de Garcés (2013:30), "de un nosotros que parte del sentido de mundo entendido como las coordenadas de nuestra actividad común, necesariamente compartida", desde la noción de interdependencia donde todos somos necesarios. Pero también de la propuesta que Laval y Dardot (2015: 660-661) hacen a partir de su análisis, donde común es descrito como:

"un principio y no en el sentido de una cosa. (...) un principio es lo que aparece primero y funda todo el resto (...) es la fuente de la que todo lo demás deriva" "es un principio político en el sentido de que ordena, impone y rige todo en la política. (...) Por político entendemos esa actividad de deliberación con la que los hombres se esfuerzan por determinar juntos lo justo, así como la decisión y la acción que proceden de esta actividad colectiva. “(...) el participar en la deliberación, la actividad de poner en común las palabras y los pensamientos".

Los grupos como la Taula de l'aigua de Terrassa plantean no sólo una lucha por el agua. A través de ella, lo que ponen de manifiesto es una lucha por un cambio de las instituciones a través de las administraciones públicas vigentes. Estas personas aspiran a mejorar, transformar y decidir sobre su entorno cotidiano, mediante una recuperación de la capacidad para poder incidir en aquellos temas que son de principal relevancia para el desarrollo de sus vidas como lo es el agua.

Este acercamiento a la temática, por parte de actores que se definen como ciudadanos/as, confiere un carácter más vinculado al espacio y las relaciones sociales que tienen lugar en el día a día y que conforman el espacio vital cotidiano. Este aspecto marca una diferencia estructural entre los primeros acercamientos por parte de grupos de cariz más técnico, ampliando el abanico de casos, inquietudes, perfiles y experiencias que surgen en todo el territorio catalán. Debido al abanico heterogéneo y de corte ciudadanista, con la presencia de nuevos actores, el abordaje se da en términos de incidencia y lucha por el común en esa reconfiguración de lo público donde lo común es de todos, y por lo tanto, debe ser gestionado entre todos, como propone Pérez Orozco $(2014)^{31}$.

Lo que aquí se analiza es un proceso incipiente que aún debe responder muchas preguntas y abrir muchos debates para la construcción de la relación común-público a través del municipalismo en las ciudades. Es decir, no se analiza un estado actual de instituciones históricas vinculadas a los bienes comunes, sino la emergencia de una institucionalidad que proclama una recuperación de los comunes, pero donde $a$

31 Amaia Pérez Orozco desarrolla en su libro Subversión feminista de la economía una propuesta de construcción de común y comunidad al que llama "Tentativa de economía diversa", partiendo del eje ecologismo social, decrecimiento y feminismo. 
priori, no existe tal vinculación; de ahí que se trate de un marco altamente dinámico y no definido. Y es en dicho proceso que hay que analizar qué elementos pasarán a formar parte de la gestión del agua entendida como bien común, cómo se experimenta dentro de los marcos del municipalismo actual para proponer otras opciones de gestión que se puedan acabar traduciendo en múltiples formas de perfil muy localizado.

Como apuntábamos, la mayoría de estos grupos nuevos no está conformada por liberados de entidades del ámbito del agua o la ecología, sino que sus miembros son habitantes de la población donde surge cada grupo. En algunos casos son voluntarios de entidades y asociaciones, en otros son vecinos y vecinas que han decidido acercarse al tema para construir un espacio de calidad, como lo es la ciudad, desde la municipalización del agua frente a una situación de crisis política e institucional. Recordando el derecho a la ciudad de Harvey (2013):

"El derecho a la ciudad es por tanto mucho más que un derecho de acceso individual o colectivo a los recursos que esta almacena o protege; es un derecho a cambiar y reinventar la ciudad de acuerdo con nuestros deseos. Es, además, un derecho más colectivo que individual, ya que la reinvención de la ciudad depende inevitablemente del ejercicio de un poder colectivo sobre el proceso de urbanización". (Harvey, 2013: 19)

Como argumentan Contreras y Chamoux (1996), para comprender las diferentes situaciones que se han producido en torno a la gestión comunal, es "necesario estudiar los mecanismos y las interacciones más o menos complejas sin limitarse a una mera descripción de las instituciones legales o consuetudinarias" ya que "se pretende aclarar las razones de la permanencia o de la desaparición de las formas comunales de organización y de gestión, así como la vigencia de un tipo u otro de derecho sobre la propiedad" (1996:12). En el caso que nos ocupa, los aportes de Contreras y Chamoux (1996) y de Laval y Dardot (2015) nos dan elementos para poder establecer el análisis de lo que acontece en el caso de la remunicipalización del agua. Primero porque aún se encuentra en una fase incipiente - sobre todo en Catalunya-, por lo cual la descripción de las instituciones legales es una fuente de información para el análisis, pero no suficiente, puesto que no se trata de un hecho instituido sino en proceso de institución. De ahí que la propuesta de Laval y Dardot (2015) de lo común como principio, expuesta antes, sea una buena herramienta para llevar a cabo un análisis más complejo que nos permita entender desde dónde se proyecta el proceso de institución.

Para Garcés (2013), la crisis no sólo tuvo afectaciones materiales, sino que puso de manifiesto los límites de la delegación y de la autonomía personal propias del liberalismo. De ahí la definición que hace del nosotros descrita antes. Las personas que se implican en este objetivo vinculan la praxis y la lucha política con formas de vida cotidiana, como reflejan los datos etnográficos, donde las personas entrevistadas quieren asumir responsabilidades en esta gestión porque entienden que les toca procurar por el bienestar colectivo, donde el municipio es entendido como el espacio común, el espacio de organización colectiva y de expresión de dicha voluntad. Este perfil es especialmente notorio en grupos como el de la ciudad de Terrassa.

La remunicipalización se abre como espacio de posibilidad para la transformación de las instituciones a partir de la creación de estructuras que amplíen su carácter 
democrático. Y es que, con la ampliación del alcance democrático de las estructuras institucionales, se abre el espacio para (re)pensar también su carácter como instituciones sociales, incorporando la importancia que deben tener los derechos humanos y sociales o el lugar que debe ocupar la ciudadanía. Por ejemplo, desde la Taula de l'Aigua de Terrassa se valora que la gestión de este servicio esté en manos directas del ente municipal, pues conlleva que los usuarios recuperen la calidad de ciudadanos, y según los mismos testimonios de este grupo: "si en esta relación son ciudadanos, pueden exigir y proponer" ${ }^{\prime 2}$. Es decir, la trascendencia recae en ocupar un espacio en la relación institución-ciudadanía para poder, desde ahí, impulsar reformas más profundas. Por defecto saben que no serán escuchados, pero les imprime carácter de legitimidad para el ejercicio de presión a la institución.

Sin embargo, no hay que perder de vista que ya dentro del marco liberal, a partir del descontento social de finales de la década de los años 1960 y 1970, según Macpherson (1977), se empezaron a introducir elementos de participación en las instituciones en lo que él describió como democracia como participación. En un contexto en el que toma presencia la dimensión municipal, cabría la posibilidad de revisar las dificultades planteadas por Macpherson y contraponerlas con las propuestas existentes en materia de participación y calidad democrática. El mismo autor también describió el modelo de la democracia como equilibrio, es decir la "democracia como competencia entre élites que produce un equilibrio sin mucha participación popular" (1977: 34). Para el autor, su insuficiencia es la que desemboca en la aparición de la democracia como participación.

Lo que vemos es que ambos modelos coexisten en el escenario actual y se corresponden con las dos tendencias expuestas al principio, entre aquellos sectores que se decantan por actitudes más individualistas y aquellos que optan por la organización y la búsqueda de lo común. Por eso no se busca sólo que el servicio sea público, sino que se formulen nuevamente los principios sobre los que se debe llevar a cabo dicha gestión asegurando la presencia de la ciudadanía como elemento activo. Se trata de analizar al final de los procesos qué elementos son incorporados en la gestión del servicio de abastecimiento domiciliario y con qué profundidad para poder determinar el alcance de transformación de la institución o si por el contrario son incorporaciones dentro del mismo. Este es el punto más complejo y también el axioma que permitiría tener elementos para analizar hasta qué punto se consiguió o no "comunalizar"33 (Harvey, 2013) las instituciones municipales de las ciudades y las políticas públicas.

\section{5. ¿Es posible hablar de la apertura hacia un nuevo marco?}

El interés por trascender las formas de gestión actuales es indicativo de los límites que definen a las instituciones en su ejercicio de las políticas públicas en relación con su compromiso con la sociedad. Podemos observar que en los distintos documentos elaborados por los colectivos, entidades y asociaciones vinculados con la temática, como

Información extraída de la reunión de la Taula de l'Aigua del día 15 de diciembre de 2015.

"En el núcleo de la práctica de comunalización se halla el principio de que la relación entre el grupo social y el aspecto del entorno considerado como bien común será a la vez colectiva y no mercantilizada, quedando fuera de los límites de la lógica del intercambio y las valoraciones de mercado" (Harvey, 2013: 115). 
el Pacto Social del agua ${ }^{34}$, se hace especial hincapié en que el agua es un bien común. Dicho documento fue impulsado a nivel estatal durante el curso 2014-2015, y en él se expone que el agua como bien común "no puede ser objeto de apropiación en beneficio de intereses privados" sino que forma "parte del patrimonio natural de la colectividad" y, como tal, "las administraciones públicas tienen la obligación de preservarla y protegerla". Para ello, la gestión se debe plantear en términos socio-ecológicos y " $100 \%$ pública o comunitaria". No "basta garantizar la gestión pública, sino que es preciso promover nuevas formas de control y de democracia participativa".

El Pacto Social por el agua nos indica la existencia de una correlación entre público y común, con una doble vertiente en relación con las instituciones públicas que acabamos de describir, que tanto busca el cambio dentro de ellas como desconfía de lo que puedan hacer o realmente cambiar. La desconfianza es señal de decepción y debilitamiento del vínculo entre institución y ciudadanía, pero aún se lucha para mantenerlo, buscando vías no exploradas. En este sentido, nos remite a lo que Laval y Dardot describen cuando exponen que "se ha puesto fin a la idea de que el Estado podría ser el recurso de la sociedad contra los efectos desastrosos del capitalismo" (Laval y Dardot, 2015: 19), en referencia al cambio substancial que representa el hecho de que la ciudadanía y los distintos movimientos busquen en la construcción del bien común una fórmula de superación del marco del cual se proviene y que nos remite a pensar en la institución como algo dinámico y que, por lo tanto puede ser modificado. Este movimiento se produce mediante mecanismos que acostumbran a situarse dentro del marco legal vigente, pero en una suerte de invención que nos muestra de forma más palpable cómo se traduce la contradicción de defensa de la institución pública a la vez que no se confía en sus mecanismos.

Si durante los años anteriores al 2011 el discurso plasmado en manifiestos, declaraciones y materiales en Catalunya se centraba en el Derecho Humano al agua, a partir de ese año irá acompañando por la idea de que el agua es un bien común:

"Y el bien común es más técnico. Bien común es una palabra que llega hace dos o tres años aquí y se abre popularmente hace nada (...) Lo asocian (las personas) a unas cosas que el mercado no podrá regular, que deben estar fuera del mercado" ${ }^{35}$

De las palabras expuestas se extrae que el cambio en la centralidad del discurso en torno al bien común coincide, por los períodos descritos, con los cambios en el perfil de los grupos locales. En un contexto de emergencia social y política el común se filtra en la sociedad atravesando el discurso generalizado que define al momento político y social, que se caracteriza por las políticas de ajuste estructural y los efectos del impacto de la crisis de 2008. Este posicionamiento, como se puede sustraer de las aportaciones de Castro (2011), es fruto de un proceso de ciudadanización del servicio de abastecimiento domiciliario que a su vez se relaciona con la asunción del derecho a la buena administración descrito por Rodríguez-Arana $(2012)^{36}$.

\footnotetext{
Pacto social por el agua, presentado públicamente en octubre de 2014.

Entrevista a EB febrero de 2015, Barcelona.

Por ello, este reconocimiento debe traducirse en un modelo sustentado y construido sobre la base del cuidado, tanto del uso del agua, como de la garantía de reparto equitativo regido por principios de justicia social. Este
} 
Con la crisis se produce un rompimiento entre ciudadanía e instituciones en su dimensión social. Frente a esta situación, la ciudadanía decide tomar cartas en el asunto y emprende la iniciativa de trabajar en dos sentidos: por una parte, exige el cumplimiento de la parte del contrato en la que era vox populi que las instituciones públicas y los órganos de gobierno tenían la obligación de procurar el bien de las personas. Por la otra, elabora propuestas de cómo, a partir de los principios que defienden, debería ser la organización social, política y económica para ajustarse a la idea de democracia compartida en el imaginario social.

Dentro de estos márgenes, la idea del bien común extendida es aquella que lo ubica como lo que es de todos y de nadie, y que por lo tanto debe ser cuidado como responsabilidad colectiva. En este sentido, podemos hablar de que hay una conciencia de cuidado, que se trata de una responsabilidad colectiva y que depende, de forma directa, de las propias personas. Este indicio muestra que la visión colectiva del elemento agua no se corresponde con la tesis expuesta por Hardin (1968) de maximización del pastizal común. Al contrario, para estos grupos la concepción del agua como bien común tiene como finalidad poner límites que se derivan del sentido de cuidado colectivo, ante un uso desmesurado llevado a cabo por un modelo de gestión que fomentó el expolio del agua en tanto que recurso.

Lo que nos encontramos en esta concepción de común en el marco urbano es una especie de propiedad colectiva - en tanto que es de todos - cuya responsabilidad es compartida, motivo por el cual se debe poder acceder a los espacios de toma de decisiones. En este sentido, observamos que los motivos que esgrimen los ciudadanos que están en estos grupos se corresponden con la concepción del ciudadano como sujeto activo que expone Rodríguez-Arana (2012). Según el autor, esto tiene que ver con el derecho fundamental a la buena administración, según el cual "la buena administración de instituciones públicas parte del derecho ciudadano a que sus asuntos comunes y colectivos estén ordenados de forma y manera que reine un ambiente de bienestar general e integral para el pueblo en su conjunto" (Rodríguez-Arana, 2012: 260). Por eso, desde esta perspectiva "han de estar conducidas por criterios de buen gobierno y buena administración" (Rodríguez-Arana, 2012). Esta respuesta parece ser una reacción a la dinámica generalizada, basada en una relación de confianza, de delegar a los representantes de las instituciones públicas el correcto mantenimiento de lo común, puesto que se partía de la idea de que las mismas tenían como finalidad el cumplimiento de dicha función social. Esta argumentación toma sentido en el marco de una comprensión según la cual la democracia y sus instituciones políticas no son propiedad de políticos o altos funcionarios, sino que son del dominio popular, de los ciudadanos (RodríguezArana, 2012).

Ahora bien, a raíz del escenario descrito, derivado de la implementación de las políticas de corte neoliberal y los efectos de la crisis de 2008, se ha desencadenado la reflexión alrededor de la importancia y la trascendencia de tomar las riendas de dicha responsabilidad. Esta actitud marca el rompimiento de lo que había sido una relación basada en la confianza y la ciudadanía como sujeto inerte (Rodríguez-Arana, 2012). Es aquí donde los grupos que pugnan por la remunicipalización de la gestión del servicio de abastecimiento de agua urbana se sitúan. Entienden y defienden que son

cambio comporta tener derecho a exigir y decidir sobre cómo, quién y a partir de qué criterios debe ser gestionada el agua municipal, puesto que es la que incide de forma directa en la calidad de vida. 
ellos quienes deben velar ${ }^{37} \mathrm{y}$ forzar una buena gestión, y que esto implica un replanteamiento del modelo.

En las luchas por la remunicipalización del agua podemos detectar elementos propios de la nueva gubernamentalidad, tales como una dinámica de interconexión social, un conjunto de innovaciones puestas en práctica que se exhiben en disputas para definir las relaciones de poder, con un protagonismo social con vocación constituyente. En este marco, los movimientos sociales son los que proponen las normas, orientaciones y dinámicas de gobierno de un espacio que está en constante disputa. La nueva gubernamentalidad se caracteriza porque es plástica y ambigua como efecto de su naturaleza vinculada a los vaivenes de la lucha política y surge como una forma de irrupción de las dinámicas sociales que cuestionan la legitimidad del neoliberalismo (Rolnik, Berardi, Mezzadra, et al., 2009).

En el caso de los grupos surgidos en Catalunya, éstos se ven legitimados para cuestionar y tomar parte ante todas aquellas incongruencias o déficits provenientes de las instituciones del espacio municipal encarnadas en la figura del ayuntamiento. Como resultado, han conseguido marcar las líneas del debate y del conflicto, erigiéndose como referentes en materia de remunicipalización, consiguiendo que los partidos -incluidos los de corte más clásico — se hayan visto forzados a aceptar parte de los puntos del Pacto Social por el agua en sus programas electorales ${ }^{38}$.

En su identificación como ciudadanos/as o grupos de ciudadanos/as, estos actores revelan una comprensión de la ciudadanía en términos inclusivos y de amplio espectro, incluso estratégico, como elemento que aglutina la diversidad, alejada de todos los aportes teóricos que han revelado el carácter excluyente que acompaña dicha categoría (Agra, 2002; Sales, 2013; Valenzuela, 2016), pero, también, de su carácter aculturador como mecanismo para la desvinculación con las informaciones propias que contienen las condiciones del lugar y red de la trama social donde los individuos se hayan situados.

En los grupos que trabajan por la recuperación de la gestión pública del agua no se observa una ruptura abrupta, sino una voluntad de garantizar el ejercicio democrático y que las personas trabajen para que la sociedad cumpla los estándares imaginados de equidad y justicia social. Lo que se observa es que el contrato social es este "sentido común", desde el cual ubican sus demandas, posicionamientos, acciones y exigencias a las instituciones políticas públicas de referencia, como lo son los ayuntamientos, cuando se trata de la gestión del servicio básico de abastecimiento de agua urbana. En este sentido, podemos decir que la transformación se sitúa dentro del marco del contrato social y en las instituciones propias del liberalismo, como describe Macpherson (1977), a través de su expresión material, de tal modo que el proceso abierto toma una apariencia suave y paulatina, que se acompaña de debate en cada paso, con el objetivo de romper y transformar para construir algo diferente aún no definido ni verbalizado, que se moldea poco a poco a partir de la reflexividad y los debates en torno a lo común. Como apuntan Laval y Dardot (2015: 21), el término común "no designa el resurgimiento de una idea comunista eterna, sino una

37 "Velaremos porque el Ayuntamiento ejerza la responsabilidad que tiene en la adopción de las decisiones oportunas que aseguren la sostenibilidad del ciclo integral del agua en la ciudad, de acuerdo con la Directiva Marco del Agua" (Pacto Social por el agua, 2014:2)

38 Como pasó con la firma por parte de la mayoría de grupos políticos de gran número de ciudades del Estado Español del Pacto Social del Agua en las elecciones municipales de 2015. 
forma nueva de oponerse al capitalismo, incluso a considerar su superación" y es el resultado de formas de lucha que están abriendo fisuras y preguntas en busca de una concreción social distinta.

También se observa que esta ruptura entre la élite política y lo que se entendía por político genera un momento que distintos autores describen como impasse (Rolnik, Berardi, Mezzadra, et al., 2009). Un espacio que se define por ser potencialmente político, pero en el que nunca se sabe qué, cómo o cuándo puede pasar. Un espacio regido por la emergencia en el que se abren múltiples líneas de pensamiento a través de las cuales se exploran y ensayan los territorios y las prácticas de otras políticas y experiencias. En el caso de la gestión municipal del agua, es constante el flujo de información de las distintas experiencias que se suceden en otras partes del mundo, de las que se aprenden y retoman aquellos elementos que se corresponden con los objetivos y los escenarios catalanes. Por este motivo, hay una proliferación de múltiples propuestas y elaboraciones. La vida se transforma en laboratorio, y los grupos que batallan la remunicipalización del agua forman parte de esta emergencia. En su trabajo cotidiano activan y experimentan herramientas, técnicas, analizan y debaten cómo se consigue lo que se proponen, combinando la cultura política de la cual se proviene con elementos adquiridos que enriquecen la capacidad de imaginar la praxis instituyente (Castoriadis, 2007). Con estos ingredientes se mezclan y conjugan otros que provienen de los movimientos sociales, tales como la transversalidad, la democracia directa, el debate y la reflexión analítica.

Por lo tanto, el campo de disputa y construcción es un espacio donde todo es posible y donde están en constante retroalimentación elementos y conceptos propios del liberalismo — como el Derecho Humano al agua_, junto con las reflexiones que en los últimos años vienen a enriquecer el debate en torno al común, los comunes, la cooperación o la colectividad, en un escenario tan definido como es el municipio.

\section{A modo de cierre}

Buscando responder a las preguntas iniciales podemos cerrar estas páginas apuntando que se observa una simultaneidad entre el surgimiento de nuevos grupos promotores de la remunicipalización en el escenario derivado del 15M y la apropiación del común como principio articulador para la recuperación de la gestión del agua.

También se aprecia que las formas empíricas que está adquiriendo el común en el ámbito específico de la gestión del sistema de abastecimiento del agua en las ciudades son principalmente fórmulas de participación social. Unas fórmulas que, en algunos casos, buscan trascender las experiencias existentes dentro de los marcos actuales, como son los procesos participativos. Este aspecto nos indica que se trata de la apuesta por lograr una participación substantiva (Castro, 2004) o real (Funes y Chávez, 2012) mediante la cual se pueda llevar a cabo la triple función de controlar, tener acceso a los datos e informaciones primarias, y estar en la posición y el lugar de poder tomar decisiones como parte implicada en la gestión.

Nos encontramos que las formas legales vigentes para llevar a cabo la gestión de servicios básicos, por parte de la administración local, no contemplan la participación de la ciudadanía, lo que permite abrir un campo, en términos legales, para 
su inclusión. El mayor obstáculo es la concepción que tanto técnicos, políticos y expertos en gestión del servicio del agua tienen de la ciudadanía y de sus capacidades para tomar decisiones de esta índole. En general, se argumenta que se trata de un campo altamente tecnificado y complejo que dificulta que las personas no expertas puedan tener el conocimiento que se requiere. A esta concepción se le agregan las fórmulas parcializadas de participación que se han ido incluyendo en la administración pública ${ }^{39}$. En este punto, la ruta pasa por hacer ejercicios dentro del marco de la legalidad, buscando combinaciones que no puedan ser desechadas por ilegales pero que se acerquen a los límites que la regulación actual permite, incorporando elementos que fomenten una democracia cada vez más cercana a la democracia directa.

También cabe recoger aquí el acento puesto en el común como principio. Ya Díaz-Polanco (2005) en su libro El elogio a la diversidad. Globalización, multiculturalismo y etnología resuelve que la única forma de superar el marco del liberalismo, desde las tesis de Kant en relación a la diversidad, pasa por entender que ésta es un metaprincipio, el metaprincipio de la diversidad, de tal modo que está antes de los principios de igualdad y libertad. De esta manera, la diversidad pasa a ser estructura y ordena todo lo que devenga en las posiciones siguientes. Del mismo modo, como ya se ha apuntado, por el carácter incipiente de la remunicipalización y la noción de común que la acompaña, resulta arriesgado hoy por hoy hablar de instituciones del común. Ahora bien, sí es posible detectar, sobre todo en el caso de Terrassa, la presencia del común como principio a partir del cual construir estas instituciones y sus formas de gestión. En este sentido, el común como principio es la concepción a partir de la cual la ciudadanía espera encontrar respuestas y soluciones para superar la situación actual, a partir de la suma de experiencias y de propuestas políticoeconómicas y sociales a lo largo de los años y las generaciones.

Esta situación abre el debate sobre los límites de la participación y sus diferencias y semejanzas con las estructuras organizativas que caracterizan la gestión comunal, y nos lleva a las preguntas planteadas por Macpherson (1977) acerca de la posibilidad de introducir cambios que aumenten la participación democrática en sociedades como la actual, puesto que la participación en sí misma no es indicativa de superación del liberalismo, sino un reajuste del mismo. Ante estas cuestiones, el estudio del servicio de abastecimiento urbano del agua se abre como un espacio idóneo para llevar a cabo este debate. Por ello, los ejes de la participación y el control social ocupan un lugar de especial relevancia, puesto que en su alcance entran todos aquellos aspectos asociados a la renovación y la búsqueda de la dimensión democrática, la transparencia, la toma de decisiones y garantizar el ejercicio del Derecho Humano como un principio básico del modelo de gestión.

En resumen, lo expuesto hace que nos interroguemos acerca de: ¿cuáles son los componentes que definen los límites del liberalismo, el ciudadanismo y los derechos, en un contexto político, económico y social a partir del cual se está buscando el modelo político, económico y social de país? ¿cuál es el papel y el alcance de la re-

39 Ecologistas en Acción elaboró un dossier sobre los mecanismos de participación contemplados en el caso del Ayuntamiento de Barcelona en el que concluye que "los representantes admiten que no hay previsto ningún acceso ciudadano a los procesos de toma de decisión sobre el modelo de gestión propiamente dicho". La participación pública en las políticas de gestión del agua en Barcelona, Annelies Broekman, coordinadora de l'Àrea d'Aigua d'Ecologistes en Acció de Catalunya i presidenta de la Xarxa per una Nova Cultura de l'Aigua, Barcelona, febrero 2012 . 
formulación de la teoría de los comunes, ahora en ámbitos urbanos? o ¿cómo validar si en efecto se está trascendiendo el marco liberal?

\section{Bibliografía}

\section{Libros y artículos:}

Aboites, Luis (1999). El agua de la nación (1888-1946). México: CIESAS.

- (2009). La decadencia del agua de la nación. Estudio sobre la desigualdad social y cambio política en México (segunda mitad s.). México: Colegio de México.

Agra, María Xosé (2002). "Ciudadanía: el debate feminista", en Quesada, F. (Ed.): Naturaleza y sentido de la ciudadanía hoy. Madrid: Publicaciones UNED, 129-160.

Bakker, Karen (2010). Privatizing water. Governance failure and the world's urban water crisis. Nueva York, Cornell University Press.

Calatayud, S. (2016). “Antes de la política hidráulica: la gestión del agua bajo el Estado liberal en España (1833-1866)", Historia Agraria, 68: 13-40.

Castoriadis, Cornelius (2007) [1975]. La institución imaginaria de la sociedad, Barcelona, Editores Tusquets.

Castro, José Esteban (2005). “Agua y gobernabilidad: entre la ideología neoliberal y la memoria histórica", Caracas: Cuadernos CENDES, vol. 22, 59: 1-21.

- (2011). "El proceso de democratización de la gestión de servicios públicos esenciales de agua y saneamiento", Hábitat y Sociedad, 2: 49-85.

Castro, J.E.; Kloster, K. y Torregora, M. (2004) "Ciudadanía y gobernabilidad en México: el caso de la conflictividad y la participación social en torno a la gestión del agua”, en El agua en México vista desde la academia, México: Academia Mexicana de Ciencias, 339-370.

Cebollada, Víctor (2014). La reforma laboral de 2012. Antecedentes, causas y consecuencias, Trabajo fin de grado, Facultad de Economía y Empresa, Universidad de Zaragoza.

Dávila, Sonia (2006). El poder del agua: ¿Participación social o empresarial? México, experiencia piloto del neoliberalismo para América Latina. México: Ítaca.

Delclòs, Jaume (2008a) "La gestió pública amb participació i control social. Cap al Dret Humà a l'aigua", en El Dret Humà a l'aigua a l'accés a l'aigua potable i el sanejament, Barcelona: Enginyeria sense fronteres, Institut dels Drets Humans a Catalunya.

- (2008b) "La gestión pública del agua con participación y control social. Hacia el derecho humano al agua", Viento Sur, 98: 84-89.

Díaz-Polanco, Héctor (2005) "Kant y la diversidad", en El elogio a la diversidad. Globalización, multiculturalismo y etnología, México: Siglo XXI, El Colegio de Sinaloa.

Díez, Xavier, Observatorio Metropolitano de Madrid, Col·lectiu Víric (2015). L'aposta municipalista. De les bullangues a les lluites actuals pels municipis lliures a Catalunya, Barcelona Editorial Virus.

Domènech, Xavier (2014). “Municipalismo y rupturas constituyentes, ¿1931:2.0?”, Sin Permiso Junio. Fecha de la consulta: febrero de 2015.

Fals Borda, Orlando (1986). Conocimiento y poder popular: Lecciones con campesinos de Nicaragua, México y Colombia, Bogotá: Siglo XXI. 
Funes, S. (2012). "Reformas y gobernanza en el sector agua", en La gobernanza del agua, un desafio actual. Hacia una mirada crítica del concepto y de su aplicación, México: IMTA.

Garcés, Marina (2013) Un mundo común. Barcelona: Bellaterra.

Gorostiza, Santiago, March, Hug y Sauri, David (2012). "Servicing Customers in Revolutionary Times: The Experience of the Collectivized Barcelona Water Company during the Spanish Civil War", Antipode 45, 4: 908-925. Doi: 10.1111/j.14678330.2012.01013.x C _2012 The Authors. Antipode C_2012 Antipode Foundation Ltd.

Hardin, Garrett (1968). "The Tragedy of the commons", Science, New Series, 162, 3859: 1243-1248.

Hastrup, Kirsten (2013). "Water and the Configuration of Social Worlds: An Anthropological Perspective", Journal of Water Resource and Protection, 5: 59-66.

Heredia, Víctor (2014). Gestión privada y municipalización en el abastecimiento en la ciudad de Málaga. El "negocio” de las Aguas de Torremolinos (1860-1930), Tesis doctoral, Departamento de Teoría e Historia Económica, Facultad de Ciencias Económicas y Empresariales, Universidad de Málaga.

Laval, Christian y Dardot, Pierre (2015). Común. Ensayo sobre la revolución en el siglo $X X I$, Barcelona: Gedisa.

Lobina, Emanuele, Kishimoto, S, y Petitjean, O.(2014). Hereto stay, Water remunicipalisation as a global trend, Amsterdam, London, Paris, Cape Town and Brussels: Transnational Institute (TNI), Public Services International Research Unit (PSIRU), Multinationals Observatory, Municipal Services Project (MSP) and Multinationals Observatory.

- (2015). Our public water future. The global experience with remunicipalisation, Amsterdam, London, Paris, Cape Town and Brussels: Transnational Institute (TNI), Public Services International Research Unit (PSIRU), Multinationals Observatory, Municipal Services Project (MSP) and the European Federation of Public Service Unions (EPSU).

López, R. y Chavez, P. (2012). "Gobernanza del agua y participación social”, en La gobernanza del agua, un desafio actual. Hacia una mirada crítica del concepto y de su aplicación, México, IMTA.

Macpherson, C.B. (1977). La democracia liberal y su época, Madrid, Alianza Editorial.

Marcet, Xavier (1986). "La Guerra Civil a Terrassa.” Terme [en línea], 1: 14-46.

Matés, Juan Manuel (2014). «La regulación del suministro de agua en España s. XIX y s. XX» Revista de Historia Industrial, 61, XXV: 15-47.

- (2016) "Las empresas concesionarias de servicios de abastecimiento de aguas potables en España (1840-1940)”. Universidad de TST, Jaén, 26: 36-67.

Pérez Orozco, Amaia (2014). Subversión feminista de la economía. Aportes para un debate sobre el conflicto capital-vida, Madrid: Traficantes de Sueños.

Sales, Tomeu (2013). "Feminismo, democracia y ciudadanía; de la crítica a la democracia patriarcal a la política democrática radical feminista", Astrolabio. Revista internacional de filosofia, 15: 72-79.

Rodríguez-Arana, Jaime (2012). «El derecho a la buena administración en las relaciones entre ciudadanos y administración pública», Anuario da Facultade de Dereito da Universidade da Coruña (AFDUC) 16: 247-273.

Rolnik, S.; Berardi, F.; Mezzadra, S. et al. (2009). Conversaciones en el impasse. Dilemas políticos del presente, Colección de mano en mano, Buenos Aires: Universidad Internacional de Andalucía Arte y Pensamiento, Ediciones Tinta Limón. 
Valenzuela, M. (2016). "Las bases epistémicas de la concepción feminista de la ciudadanía" Estudios Feministas, 24(1): 31-43.

Páginas web:

Aigua és Vida (AeV): http//www.aieguesvida.org (fecha de consulta: marzo, 2015). Agència Catalana de l'Aigua (ACA): http://aca-web.gencat.cat/aca/appmanager/aca/aca? $\mathrm{nfpb}=$ true\&_pageLabel=P1210054461208200724644 (fecha de consulta: mayo, 2016). Asociación Española de Operadores Públicos de Abastecimiento y Saneamiento (AEOPAS): http://www.aeopas.org/ (fecha de consulta: mayo 2016).

Consorci per a la gestió d'Aigües de Catalunya (CONGIAC): http://www.congiac.com/es/ (fecha de consulta: mayo 2016).

Institut d'Estadística de Catalunya (IDESCAT): http://www.idescat.cat/

Plan B Europa: http://planbeuropa.es/

\section{Informes:}

Vallvé, Xavier (2016). Informe Pobresa i privació de subministraments bàsics. 'Pobresa energètica a Catalunya, una qüestió d'ingressos, Entitats Catalanes d'Acció Social (ECAS).

Balasch, Laia (2012). “Agbar, una gestió il·legítima de l'aigua”, Anuari mèdia.cat.

Carrillo, Miquel (2015) “Agbar no pensa informar-te d'això”, Crític http:/www.elcritic.cat/ blogs/sentitcritic/2014/07/15/agbar-no-pensa-informar-te-daixo/ Fecha de consulta: 2 septiembre 2016

Expansión Barcelona (2016). «Cataluña: El TSJC anula la concesión del agua a la empresa mixta del AMB, Agbar y La Caixa», Expansión, 16/03/2016.

Actividades y jornadas:

Lobina; Emanuele (2016). «La remunicipalización llegó para quedarse», Encuentro de ciudades por el agua pública, 3 y 4 noviembre 2016, Ayuntamiento de Madrid. 